\title{
Protective effects of polyunsatutared fatty acids supplementation against testicular damage induced by intermittent hypobaric hypoxia in rats
}

Rodrigo L Castillo ${ }^{1}$, Andrea B Zepeda ${ }^{2,3}$, Stefania E Short ${ }^{2}$, Elías Figueroa ${ }^{2,4}$, Eduardo Bustos-Obregon ${ }^{5}$ and Jorge G Farías ${ }^{2^{*}}$

\begin{abstract}
Background: Intermittent hypobaric hypoxia $(\mathrm{HH})$ induces changes in the redox status and structure in rat testis. These effects may be present in people at high altitudes, such as athletes and miners. Polyunsaturated fatty acids (PUFA) can be effective in counteracting these oxidative modifications due to their antioxidants properties. The aim of the work was to test whether PUFA supplementation attenuates oxidative damage in testis by reinforcing the antioxidant defense system. The animals were divided into four groups (7 rats per group): normobaric normoxia ( 750 tor; pO2 $156 \mathrm{mmHg}$; Nx); Nx + PUFA, supplemented with PUFA (DHA: EPA = 3:1; $0.3 \mathrm{~g} \mathrm{~kg}^{-1}$ of body weight per day); hypoxic hypoxia ( 428 tor; pO2 $90 \mathrm{mmHg} ; \mathrm{Hx}$ ) and, $\mathrm{Hx}+$ PUFA. The hypoxic groups were exposed in 4 cycles to $96 \mathrm{~h}$ of $\mathrm{HH}$ followed by $96 \mathrm{~h}$ of normobaric normoxia for 32 days. Total antioxidant capacity (FRAP) and lipid peroxidation (malondialdehyde, MDA) in plasma and reduced (GSH)/oxidized glutathione (GSSG) ratio, tissue lipid peroxidation (TBARS) and antioxidant enzymes activity were assessed at the end of the study in testis. Also, SIRTUIN 1 and HIF-1 protein expression in testis were determined.
\end{abstract}

Results: $I H H$ increased lipid peroxidation in plasma and HIF-1 protein levels in testis. In addition, IHH reduced FRAP levels in plasma, antioxidant enzymes activities and SIRTUIN 1 protein levels in testis. PUFA supplementation attenuated these effects, inducing the increases in FRAP, in the antioxidant enzymes activity and HIF-1 levels.

Conclusions: These results suggest that the $\mathrm{IH}$ model induces a prooxidant status in plasma and testis. The molecular protective effect of PUFA may involve the induction of an antioxidant mechanism.

Keywords: Intermittent hypoxia, Testis, Oxidative stress, PUFA, SIRTUIN 1

\section{Background}

Humans at high altitudes are exposed to hypobaric hypoxia, a condition where reduced barometric pressure decreases oxygen availability [1] resulting in lower circulating oxygen levels [2]. Populations living at high altitudes are exposed to chronic hypobaric hypoxia whereas activities such as mountain sports, tourism and certain-jobs (i.e. customs agents, mining, educational and health workers) performed in areas over 1,500 meters above sea level (asl) involve intermittent exposure to hypobaric hypoxia (IHH) [3-5].

\footnotetext{
* Correspondence: jorge.farias@ufrontera.cl

${ }^{2}$ Departamento de Ingeniería Química, Facultad de Ingeniería y Ciencias, Universidad de La Frontera, Casilla 54-D, Temuco, Chile

Full list of author information is available at the end of the article
}

At high altitudes, oxidative stress is higher than at sea level under normobaric conditions, due to a combination of factors such as temperature fluctuation, increased UV light intensity and metabolic rate, low blood oxygenation and localized anoxia/reoxygenation [6]. Due to their ubiquitous nature, increased reactive oxygen species (ROS) may cause cell membrane damage through lipid peroxidation, protein oxidation, DNA damage, and consecutively impaired ATP production $[7,8]$. Oxidative stress may also produce cell swelling, decrease cell membrane fluidity, prevent the maintenance of ionic gradients and lead to tissue inflammation, which can result in structural and functional detrimental effects [6]. Chronic and IHH have been associated with an increase of oxidative damage in 
testis [9]. IHH induces higher levels of lipid peroxidation, reduces glutathione reductase activity, and decreases the epididymal sperm count [10]. These changes have been partially attenuated by the supplementation of antioxidants such as melatonin and ascorbate [10,11].

Studies and clinical intervention trials have demonstrated a probable protective role of dietary fish oils and n-3 polyunsaturated fatty acids (PUFA) in cardiovascular diseases [12-14]. The beneficial effects have been attributed to the improvement of vascular endothelial cell function, enhancement of vascular reactivity and compliance, modulation of lipid metabolism and reduction of inflammatory cytokine production [15]. Docosahexaenoic acid (DHA, C22:6n-3), a major n-3 PUFA, is generally acknowledged as a physiologically active component of these effects [16]. The cellular and molecular mechanisms underlying the beneficial effects of DHA on various cell types during ischemia-reperfusion (IR) injury, especially cardiomyocytes, vascular endothelial, and smooth muscle cells, have been extensively studied $[17,18]$. These protective mechanisms include the induction anti-inflammatory transcriptional pathways, the decrease in intracellular $\mathrm{Ca}^{2+}$ levels, the suppression of vascular proliferation, and the improvement of cell membrane integrity $[19,20]$. Indeed, PUFA may induce an antioxidant mechanism through the induction of nuclear factor erythroid 2-related factor 2 (Nrf2), a master transcriptional factor for antioxidant genes and glutathione synthesis in vascular tissue [21]. In testis, PUFA may be beneficial for improving sperm motility and viability following acute doxorubicin-induced injury [22]. However, the effects of PUFA on oxidative stress markers and antioxidant mechanisms have not been well characterized in testis.

Therefore, in an established rat model of IHH we hypothesized that PUFA supplementation attenuates oxidative damage in testis by reinforcing the antioxidant defense system.

\section{Methods}

\section{Experimental design}

All animal care and procedures complied with the principles of animal care outlined by the National Society Laboratory and the Medical Research, and the Guide for the Care and Use of Laboratory Animals (Institute of Animal Laboratory Resources, 1996), and were approved by the Faculty of Medicine's Bioethics Committee. (Study number: CBA\# 0627 FMUCH). All experiments were performed in adult male Wistar rats $(n=28)$. The animals were randomly divided into four equal groups: normobaric normoxia ( 750 tor; pO2 $156 \mathrm{mmHg}$; Nx, $\mathrm{n}=7) ; \mathrm{Nx}+$ PUFA $(\mathrm{n}=7)$, supplemented with PUFA (DHA: $\mathrm{EPA}=3: 1 ; 0.3 \mathrm{gkg}^{-1} \mathrm{~d}^{-1}$ ) via gavage; hypoxic hypoxia ( 428 tor; pO2 $90 \mathrm{mmHg} ; \mathrm{Hx}, \mathrm{n}=7$ ) and, Hx + PUFA $(n=7)$. The hypoxic groups were exposed in 4 cycles to $96 \mathrm{~h}$ of hypobaric hypoxia followed by $96 \mathrm{~h}$ of normobaric normoxia for 32 days. The desired pressure inside the hypobaric chamber was achieved by pressure changes simulating altitude increases of $150 \mathrm{~m}$ per minute. The animals in the $\mathrm{Nx}$ groups were lodged in the same room as the $\mathrm{Hx}\left(22^{\circ} \mathrm{C}\right)$. The 4 groups received the same amount of daily food and drinking water (15 $\mathrm{g}$ of standard pellet meals; water ad libitum). Body weight was determined at the beginning of the study and then every other day, and at the end of each period. The PUFA supplementation was maintained for the 32 days of the study. At the end of the study and after blood samples and tissue extraction, the rats were euthanized by cervical dislocation in a room separated from the remaining animals.

\section{Blood sample preparation}

Animals from both groups were injected intraperitoneally with $0.2 \mathrm{ml}$ of ketamine to numb them; subsequently the cardiac puncture was performed using the Burhoe's Method modified, in accordance with the handbook "The Rat in Laboratory Investigation". One $\mathrm{ml}$ of blood was collected from each and centrifuged (Centrifuge 5804R, Eppendorf AG, No. 5805XK634757) at $489 \times$ $g$ for $35 \mathrm{~min}$ to separate plasma from blood cells. The plasma was separated in $100 \mu \mathrm{l}$ aliquots and kept at $-80^{\circ} \mathrm{C}$ until use.

\section{Determination of antioxidant capacity and lipid peroxidation in plasma}

Plasma antioxidant capacity was assessed by measuring the ability of plasma compounds to reduce ferric $\left(\mathrm{Fe}^{+3}\right)$ to ferrous iron $\left(\mathrm{Fe}^{+2}\right.$ ) (Ferric Reducing Ability of Plasma assay, FRAP). The $\mathrm{Fe}^{+2}$-III/TPTZ-complex was detected at light absorbance $594 \mathrm{~mm}$ (spectrophotometer method), with a detection limit of $10 \mu \mathrm{mol} / \mathrm{l}$ [23]. Lipid peroxidation was measured by determining of malondialdehyde (MDA), in plasma samples by high performance liquid chromatography [24]. The results were expressed as $\mu \mathrm{mol} / \mathrm{mg}$ protein.

\section{Assessment of redox status in testis}

The testes were homogenized in $0.5 \mathrm{~mL}$ of extraction buffer (Tris 50 mM, NaCl 100 mM, EDTA 1 mM, EGTA $2.5 \mathrm{mM}$, Tween-20 0.1\% [pH 7.4], phenylmethylsulfonyl fluoride [PMSF] $100 \mu \mathrm{g} / \mathrm{mL}$; Sigma-Aldrich) with a Potter homogenizer (Glass-Col K4424; Glas Col, Terre Haute, Indiana) at $.050301 \times g$. The samples were then centrifuged at $7820 \times g$ for 30 minutes at $4{ }^{\circ} \mathrm{C}$.

The $\mathrm{Cu}-\mathrm{Zn}$ SOD assay is based on the SOD-mediated increase in the rate of autooxidation of catechols in an aqueous alkaline solution in order to yield a chromophore with a maximum absorbance of $525 \mathrm{~nm}$ [25]. One $\mathrm{Cu}-\mathrm{Zn} \mathrm{SOD}$ unit is defined as the activity 
that doubles the auto-oxidation background, and the results are expressed as units/mg of protein. Soluble GSHPx activity was measured spectrophotometrically in the cytosolic fraction (100000 g supernatant) by the reduction of glutathione disulfide coupled to NADPH oxidation by glutathione reductase [26]. One GSH-Px unit is defined as the activity that oxidizes $1 \mu \mathrm{mol}$ of $\mathrm{NADPH} / \mathrm{min}$ and is expressed as units/mg of protein.

The redox status in testicular tissue was assessed by a fluorometric method in order to measure oxidized (GSSG) and reduced glutathione (GSH). The o-phthalaldehyde (OPT) was used as a fluorescent reagent. This method assesses the reaction of GSH with OPT at $\mathrm{pH} 8$ and of GSSG with OPT at pH 12; GSH can be complexed to N-ethylmaleimide to prevent interference of GSH with the measurement of GSSG [27]. The GSHGSSG ratio was then calculated. Lipid peroxidation was assessed by the thiobarbituric acid reaction at $\mathrm{pH} 3.5$, followed by solvent extraction with a mixture of $\mathrm{n}$-butanol/pyridine $(15: 1, \mathrm{v} / \mathrm{v})$ [28]. Tetramethoxypropane was used as the external standard, and the levels of lipid peroxides were detected spectrophotometrically at $532 \mathrm{~nm}$ and expressed as mmol TBARS (Thiobarbituric acid reactive substances)/ mg protein.

\section{SIRTUIN 1 and HIF-1a expression determined by Western Blot (SDS/PAGE)}

Aliquots of testis tissue homogenate of testis containing an equal concentration of proteins, $50 \mu \mathrm{g}$, were electrophoresed $(100 \mathrm{~V})$ on a $12 \%$ SDS/PAGE gel as previously described by Farias et al. [10], using a primary mouse SIRTUIN 1 antibody against rat (\#8469; 1: 250 dilution) (Cell Signaling, Inc., MA, USA), a primary rabbit HIF- $1 \alpha$ (sc-10790; 1:250 dilution) (Santa Cruz Biotechnology, Santa Cruz, CA, USA), a secondary anti-mouse (\#7076; 1:1000) (Cell Signaling, Inc., MA, USA) and a secondary anti-rabbit antibody (IC-3R01; 1:1000) (Imuny Rheabiotech, SP, Brazil). Finally, a primary mouse $\beta$-tubulin antibody (\#3873; 1: 500) (Cell Signaling, Inc., MA, USA) was used as a loading control in all Western blot assays. The bands obtained were analyzed with ImageJ software (http://imagej.nih.gov/ij/) and the integrated density values of the glutathione reductase bands were normalized by dividing by the value of the loading control band.

\section{Statistical analysis}

The Shapiro-Wilk test was used for checking normal distribution of data. The results were presented as mean \pm standard deviation (SD). Data were analyzed using the one-way analysis of variance (ANOVA) test followed by Bonferroni analysis. The level of statistical significance was set at $\mathrm{p}<0.05$ for all tests.

\section{Results}

PUFA supplementation attenuates pro-oxidant imbalance induced by IHH in plasma

After the IHH cycles, FRAP levels show a lower levels than the Nx group $(199 \pm 39.8$ vs $276.7 \pm 63.4 \mu \mathrm{mol}$ $\mathrm{Fe}-\mathrm{II} / \mathrm{L})(\mathrm{P}<0.05)$. PUFA supplementation induces an improvement of antioxidant capacity, showing higher levels of FRAP in Nx + PUFA $(362.8 \pm 32.1 \mu \mathrm{mol} \mathrm{Fe-II/L})$ and $\mathrm{Hx}+\mathrm{PUFA} \quad(378.3 \pm 44.8 \mu \mathrm{mol} \quad \mathrm{Fe}-\mathrm{II} / \mathrm{L}) \quad(\mathrm{P}<0.05)$ (Figure 1A). The MDA show a higher levels than the values obtained in the plasma of the $\mathrm{Nx}$ group $(0.54 \pm$ 0.13 vs. $0.39 \pm 0.11 \mu \mathrm{mol} \mathrm{MDA} / \mathrm{mg}$ prot. $)(\mathrm{P}<0.05)$. PUFA supplementation attenuates these changes in the $\mathrm{Nx}$ group $(0.29 \pm 0.15 \mathrm{MDA} / \mathrm{mg}$ prot.) and maintains these values inclusive after $\mathrm{IHH}$ exposure $(0.37 \pm 0.10$ $\mathrm{MDA} / \mathrm{mg}$ prot.) $(\mathrm{P}<0.05)$ (Figure $1 \mathrm{~B})$. No significant differences were found between MDA levels in the PUFA group at baseline and following ischemia-reperfusion (Figure 1A-B).

\section{PUFA supplementation increases antioxidant enzymes activity in testis of rats subjected to $\mathrm{IHH}$}

The SOD and GSH-Px showed lower levels of activity in testis of $\mathrm{Hx}$ rats than in the with $\mathrm{Nx}$ group (SOD, 10.9 \pm 3.23 vs. $7.24 \pm 1.48 \mathrm{U} / \mathrm{mg}$ prot.; GSH-Px, $0.029 \pm 0.007$ vs. $0.013 \pm 0.006 \mathrm{U} / \mathrm{mg}$ prot. $)(\mathrm{P}<0.05)$. After PUFA supplementation, both the $\mathrm{Nx}$ and $\mathrm{Hx}$ groups showed higher SOD and GSH-Px activity than the non-supplemented rats (SOD, Nx: $17.66 \pm 2.5 \mathrm{U} / \mathrm{mg}$ prot. and $\mathrm{Hx}$ : $12.57 \pm$ $1.4 \mathrm{U} / \mathrm{mg}$ prot.) (GSH-PX, Nx: $0.049 \pm 0.009 \mathrm{U} / \mathrm{mg}$ prot. and $\mathrm{Hx}: 0.034 \pm 0.005 \mathrm{U} / \mathrm{mg}$ prot. $)(\mathrm{P}<0.05)$ (Figure 2A-B).

PUFA supplementation attenuates pro-oxidant damage in testis of rats subjected to $\mathrm{IHH}$

Lipid peroxidation in testis of $\mathrm{Nx}+$ PUFA rats, represented by TBARS levels, was significantly lower compared with levels in the $\mathrm{Nx}$ group without supplementation $(6.10 \pm 1.70$ vs. $2.90 \pm 0.90 \mathrm{nmol}$ TBARS/mg prot. $)(\mathrm{P}<$ $0.05)$. Indeed, IHH induced higher levels of TBARS in testis than those values obtained from testis samples from Hx rats supplemented by PUFA $(10.7 \pm 1.7$ vs. $6.63 \pm$ $1.4 \mathrm{nmol}$ TBARS/mg prot.) $(\mathrm{P}<0.05)$ (Figure $3 \mathrm{~A})$.

The GSH/GSSG ratio, as a marker of the intracellular antioxidant status, is shown in Figure 3B. The $\mathrm{Nx}+$ PUFA group shows higher levels of GSH/GSSG ratio in testis than the Nx group $(48.5 \pm 9.8$ vs $32.3 \pm 11.2)(\mathrm{P}<$ 0.05). In the Hx group the values of this ratio were lower than the Nx group $(19.7 \pm 7.8)(\mathrm{P}<0.05)$. PUFA supplementation in $\mathrm{Hx}$ rats showed higher values of GSH/ GSSG than in the Hx rats $(37.4 \pm 6.2)(\mathrm{P}<0.05)$. PUFA administration did not affect the SIRTUIN-1 and HIF-1a levels in the Nx group. 

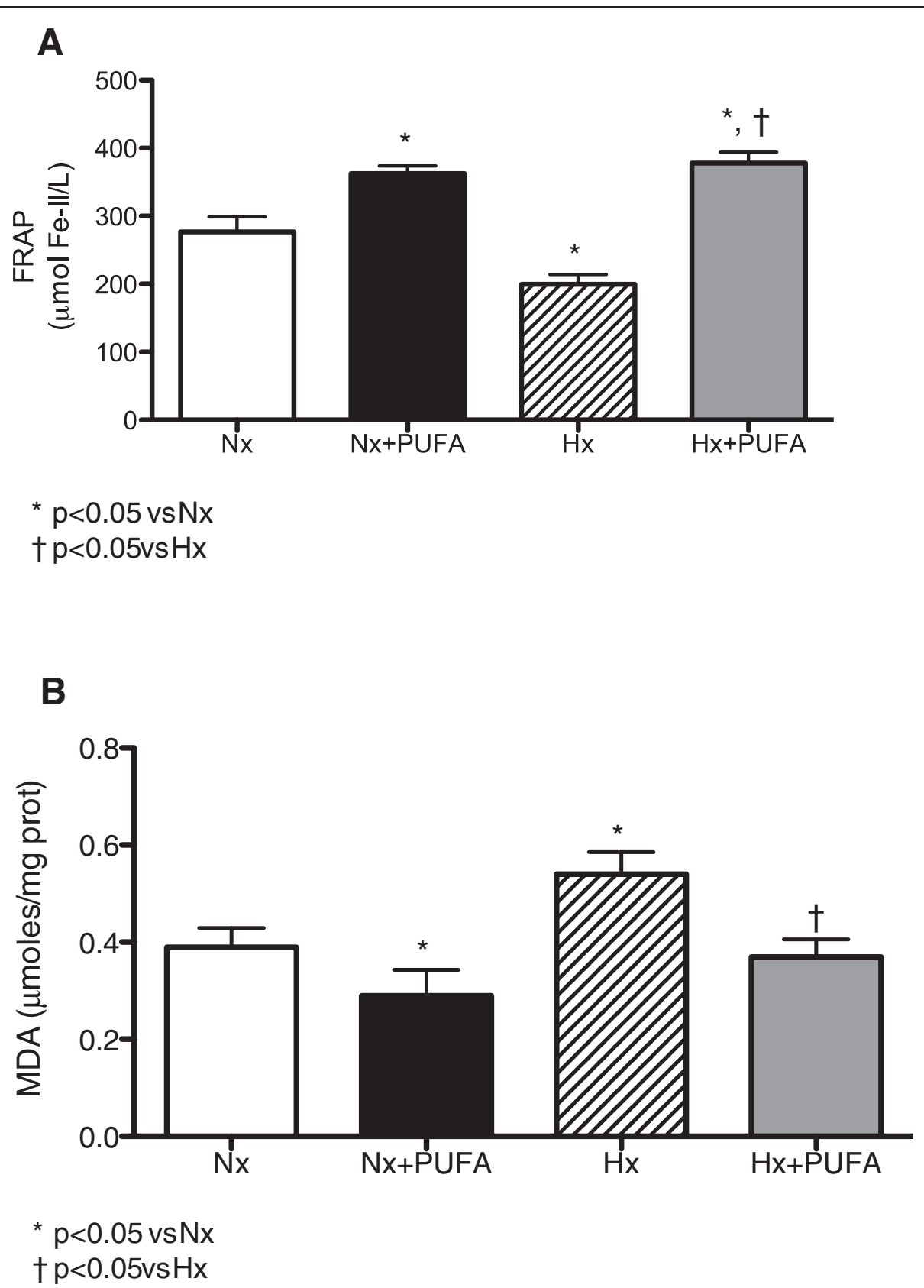

Figure 1 Effects of PUFA supplementation on antioxidant capacity and lipid peroxidation in plasma. FRAP levels (A) and malondialdehyde (MDA) (B) levels were assessed in plasma at the end of study. Normobaric $(N x, n=7)$ and intermittent hypobaric hypoxia $(H x, n=7)$. Supplemented rats: normobaric, Nx+PUFA $(n=7)$ and intermittent hypobaric hypoxia, $\mathrm{Hx}+$ PUFA $(n=7)$. Bars indicate mean \pm SD. Significant differences: ${ }^{*} p<0.05$ vs $\mathrm{Nx}^{+}{ }^{\dagger} \mathrm{p}<0.05$ vs $\mathrm{Hx}$.

PUFA supplementation induces changes of HIF-1a and SIRTUIN-1 levels in testis after IHH

No significant differences in HIF- $1 \alpha$ and SIRTUIN 1 testis levels were found between $\mathrm{Nx}$ and $\mathrm{Nx}+$ PUFA rats. As illustrated in Figure 4, higher levels of HIF-1 and lower levels of SIRTUIN-1 compared to the $\mathrm{Nx}$ group were determined $(\mathrm{P}<0.05)$. PUFA administration showed no significant difference in SIRTUIN-1 and HIF- $1 \alpha$ levels obtained in the $\mathrm{Nx}$ and $\mathrm{Nx}+$ PUFA groups, respectively.

\section{Discussion}

Our results demonstrate that the $\mathrm{IHH}$ model induced a prooxidant status in the plasma and testis of rats. The oxidative damage was attenuated by chronic supplementation with omega-3 fatty acids. 


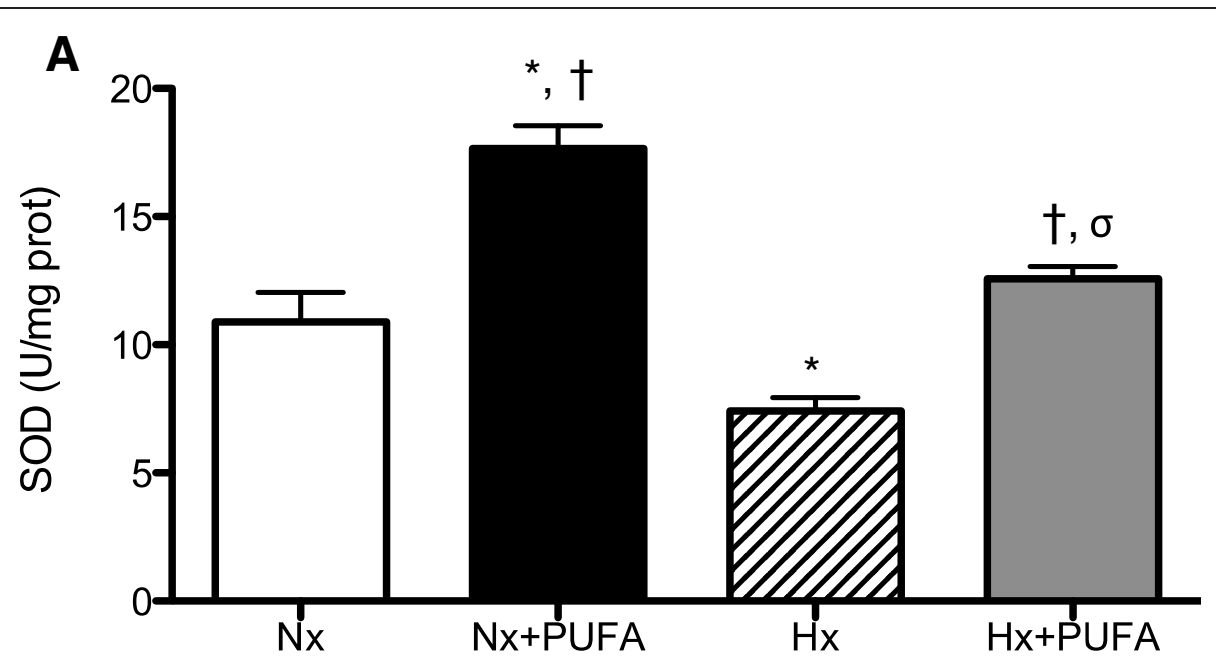

${ }^{*} p<0.05 \mathrm{vsNx}$

$+\mathrm{p}<0.05 \mathrm{vsHx}$

$\sigma p<0.05 v s \mathrm{Nx}+\mathrm{PUFA}$

B

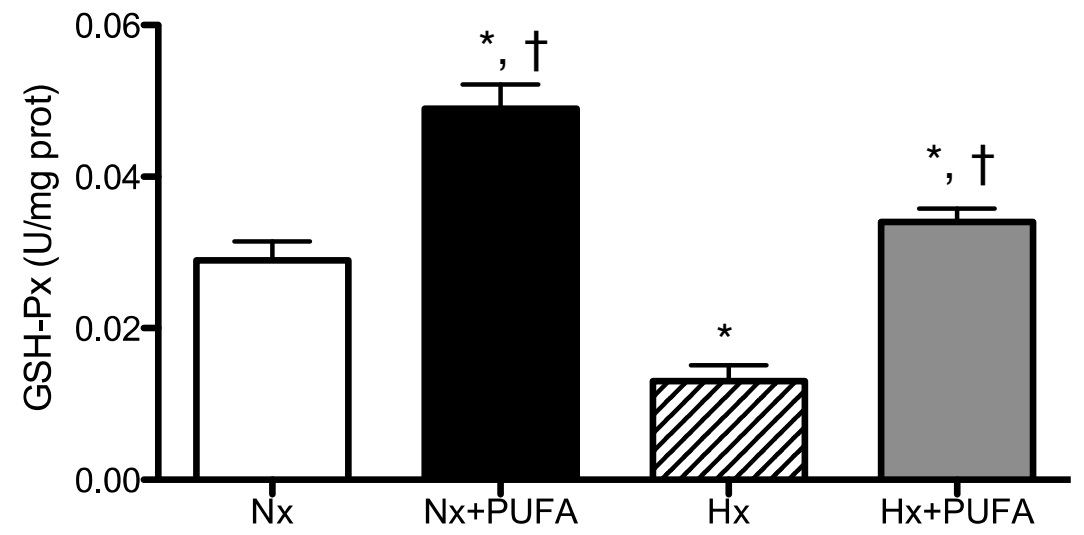

${ }^{*} \mathrm{p}<0.05 \mathrm{vsNx}$

$\dagger p<0.05 v \mathrm{sH}$

Figure 2 Effects of PUFA supplementation on antioxidant enzymes activity in testis. Superoxide dismutase (SOD, A) and glutathione peroxidase (GSH-Px, B) activities were assessed in testis at the end of study. Normobaric $(\mathrm{Nx}, \mathrm{n}=7)$ and intermittent hypobaric hypoxia $(\mathrm{Hx}, \mathrm{n}=7)$. Supplemented rats: normobaric, Nx + PUFA $(n=7)$ and intermittent hypobaric hypoxia, Hx + PUFA $(n=7)$. Bars indicate mean \pm SD. Significant differences: ${ }^{*} p<0.05$ vs Nx; ${ }^{\dagger} p<0.05$ vs Hx; ${ }^{\circ} p<0.05$ vs Nx+PUFA.

The molecular mechanism of the protective effect of PUFA may involve the induction of antioxidant effects.

The determinations of the time course of oxidative stress markers are important for the study of clinical models associated with $\mathrm{IHH}$, such as pulmonary hypertension and obstructive sleep apnea [29]. Increases in oxidative stress parameters have been demonstrated in a model of shorter human hypoxia exposure, such as in vivo lipid peroxidation products (i.e. F2-isoprostanes) and systemic oxidative stress [30,31]. Indeed, antioxidant capacity, assessed through reducing ability of plasma (FRAP) levels has been lower in acute hypoxic exposure compared with normobaric conditions in sports $[32,33]$. Also, this prooxidant state 

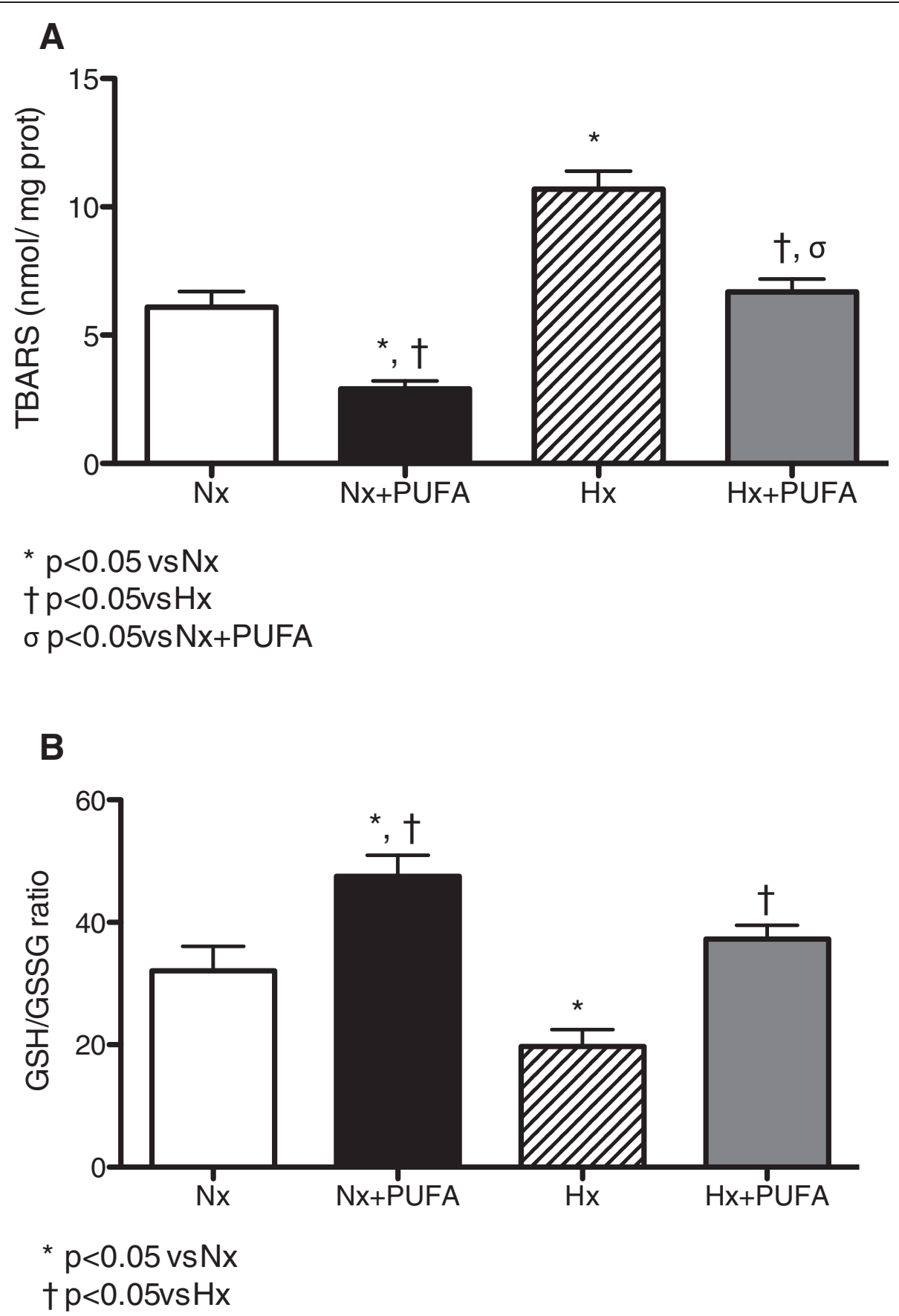

Figure 3 Effects of PUFA supplementation on oxidative stress markers in testis. TBARS levels (A) and (GSH/GSSG) ratio (B) were assessed in testis at the end of study. Normobaric $(N x, n=7)$ and intermittent hypobaric hypoxia $(H x, n=7)$. Supplemented rats: normobaric, Nx+PUFA $(n=7)$ and intermittent hypobaric hypoxia, Hx +PUFA $(n=7)$. Bars indicate mean \pm SD. Significant differences: ${ }^{*} p<0.05$ vs $\mathrm{Nx}^{+} \mathrm{p}<0.05$ vs $\mathrm{Hx}$; ${ }^{\sigma} \mathrm{p}<0.05$ vs Nx+PUFA.

induced by $\mathrm{IHH}$ has been linked to oxidative stress occurrence in local tissues in rats, such as brain, heart, lung and kidney [4,34]. In our study, chronic IHH caused lower FRAP levels (Figure 1A) and higher MDA levels (Figure 1B) in the plasma, these redox changes are consistent with the prooxidant systemic effects induced by our model of IHH. However, the effects of prooxidant imbalance associated with $\mathrm{IHH}$ in testis are poorly characterized.

Male Wistar rats have responded to chronic hypoxia with blood and tissue changes, resembling the human response to high altitudes, including polyglobulia and 


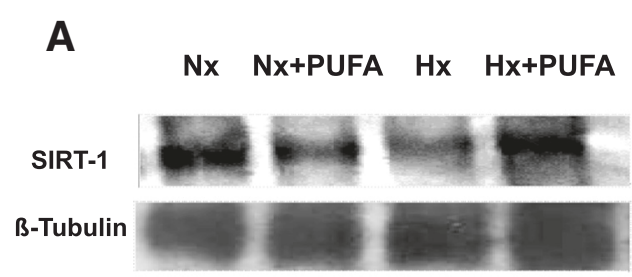

B

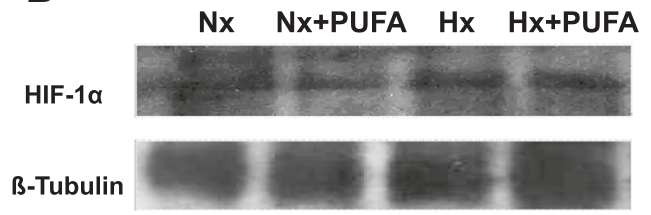

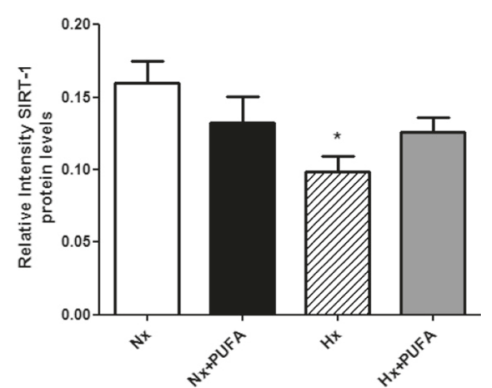

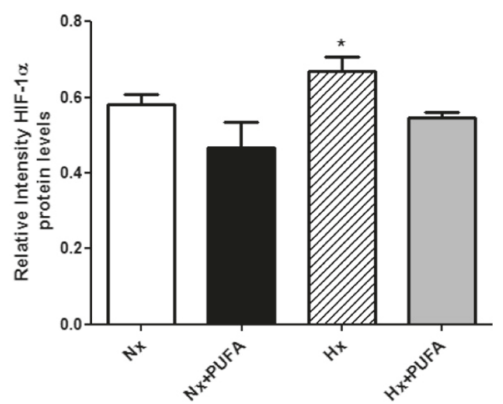

Figure 4 Effects of PUFA supplementation on SIRTUIN-1 and HIF-1. Protein Expression Level. A: Sirtuin-1 (SIRT-1) and B: HIF-1a levels were assessed in testis at the end of study. Normobaric $(N x, n=7)$ and intermittent hypobaric hypoxia $(H x, n=7)$. Supplemented rats: normobaric, $\mathrm{Nx}+$ PUFA $(n=7)$ and intermittent hypobaric hypoxia, Hx + PUFA $(n=7)$. Bars indicate mean \pm SD. Significant differences: ${ }^{*} p<0.05$ vs Nx.

right ventricular hypertrophy [35]. IHH leads to a decrease in the testicular mass, an increase in the interstitial space, and a reduction of the seminal epithelium $[9,36]$. Recent works have reinforced the existence of an oxidative metabolism in the epididymis of rats subjected to hypobaric hypoxia due to the increased in the regulator enzyme expression of ROS. This rise in ROS production induces an high rate of apoptosis at germinal cell level, leading to a state of hypo-spermatogenesis that may jeopardise masculine fertility [36]. In our study, the vulnerability of male reproductive tissue to oxidative challenges caused by IHH may depend on antioxidant system activity, e.g. the glutathione pathways (glutathione reductase activity) [10]. With regard to these findings, our results are consistent with the tissue prooxidant state, demonstrated by the lower levels of SOD, GSH-Px and GSH/GSSG ratio and the higher levels of TBARS determined in testis of rats subjected to IHH compared with Nx group (Figures 2 and 3).

The hypoxia-induced transcriptional responses are highly regulated in normal development and are deregulated in many diseases [37]. HIF-1 is formed by two subunits: HIF- $1 \alpha$ and HIF- $1 \beta$ have been identified as proteins containing a basic helix-buckle-helix and a Per/ARNT/Sim (PAS) domain, which are responsible for the hypoxic induction of erythropoietin. Under reduced oxygen levels, these two proteins heterodimerize in the nucleus and induce the transcription of genes involved in angiogenesis, metabolic and hypoxic adaptation and resistance to oxidative stress and increase invasive properties [38]. In ischemia induced by hypobaric hypoxia, HIF- $1 \alpha$ transcriptionally activates hundreds of genes vital for cell homeostasis and angiogenesis. Although potentially beneficial in ischemia, the upregulation of the HIF- $1 \alpha$ transcription factor has been linked to inflammation and oxidative stress occurrence [39]. In agreement with this, our results demonstrated higher levels of HIF- $1 \alpha$ in testis of rats subjected to IHH compared with the Nx group (Figure 4).

Recent reports have demonstrated that PUFA are beneficial against structural brain and cardiac IR injury $[40,41]$. Although the protective mechanisms associated with PUFA supplementation are not fully understood, some have suggested that PUFA protection is primarily via anti-inflammatory effects, such as inhibition of monocyte infiltration, NF-kappaB activation and increased biosynthesis of anti-inflammatory active compounds (resolvins-derived from PUFA metabolism) [42]. On another hand, the induction of antioxidant defensive mechanisms is related to direct effects exerted on the cell membrane and induction of genomic responses. This antioxidant activity may act as a homeostatic mechanism counteracting the increased oxidative stress induced by $\mathrm{IHH}$, such as the induction of antioxidant and detoxifying activities [43]. Our results showed that PUFA administration could effectively counteract prooxidant damage, 
mediated by the attenuation of systemic and tissue oxidative stress induced by IHH. This is reflected by an increase SOD and GSH-Px activities (Figure 2A-B), a reduction in lipid peroxidation and increased FRAP values (Figure 1A). These effects obtained through pharmacological doses (supplementation), confirm the helpful role of PUFA as a promising antioxidant against $\mathrm{IHH}$; however, the type of antioxidant effect, for example scavenger or probable antioxidant compound induction, has not been clearly described.

In the current study, we endeavored to explore some molecular pathways related to the induction of antioxidant responses. SIRTUIN family proteins are involved in specific aspects of metabolism and ROS scavenging proteins, and SIRTUIN-1 and SIRTUIN-3 in particular, have been exhibited effects on antioxidant enzymes activity [44]. Some works show that the upregulation of SIRT1 suppresses the expression levels of pro-inflammatory cytokines and increases the expression levels of SOD and catalase in astrocytes, skeletal muscle and cardiomyocyte, thereby inducing cell protection $[45,46]$. Experimental evidence suggested that PUFA exert anti-inflammatory functions in both human and animal models by increasing the expression, phosphorylation and activity of the AMPK pathway, which further leads to SIRT1 overexpression $[47,48]$. However, the antioxidant effects are not well characterized. In our study, no significant differences were found following PUFA administration in the Nx group, and it may be that the dose used $\left(0.3 \mathrm{gkg}^{-1} \mathrm{~d}^{-1}\right)$ was lower than the dose at which exert antioxidant effects in other organs, such as heart, brain and intestinal rat tissues [48,49]. Moreover, the incorporation of these fatty acids into biological membranes was not measured in this study, a significant factor in determining the protective effect against oxidative stress, for example DHA [50,51]. However, the role of other transcriptional pathways cannot be ruled out, for example of Nrf2 dependent signaling pathways. Indeed, our data showed lower levels of SIRTUIN-1 protein levels in IHH rat testis, effects reversed by PUFA supplementation. This would account for a probable mechanism induced by hypoxic insult of short-term not associated with the preconditioning effect [52] (Figure 4).

\section{Conclusions}

In summary, we have concluded that IHH induces a pro-oxidant state in plasma and rat testes through increased lipid peroxidation and reduced in antioxidant mechanisms. PUFA supplementation attenuated these changes by inducing the enzymatic and non-enzymatic antioxidant defense systems. This decreases the possibility of structural and functional damage that need to be characterized. The molecular mechanisms induced by
PUFA and IHH in rat testis, therefore should be determined in greater detail.

\section{Competing interests}

The authors have declared that they have no competing interest.

\section{Authors' contributions}

RLC and JGF participated in planning research. All the authors participated in performing experiments, and were responsible for data interpretation and collaborate in writing process of the manuscript. All authors read and approved the final manuscript.

\section{Acknowledgments}

The authors are thankful for support provided by FONDECYT 11110426 (R.L. Castillo), CONICYT doctoral fellowship to A. Zepeda and E. Figueroa, DIUFRO grant DI13-2014.

\section{Author details}

${ }^{1}$ Pathophysiology Program, Institute of Biomedical Sciences, Faculty of Medicine, University of Chile, Santiago, Chile. ${ }^{2}$ Departamento de Ingeniería Química, Facultad de Ingeniería y Ciencias, Universidad de La Frontera, Casilla 54-D, Temuco, Chile. ${ }^{3}$ Department of Biochemical and Pharmaceutical Technology, School of Pharmaceutical Sciences, University of São Paulo, Av. Prof. Lineu Prestes, 580 - Bloco 16, São Paulo, SP 05508-900, Brazil. ${ }^{4}$ School of Aquaculture, Catholic University of Temuco, Faculty of Natural Resources, Temuco, Chile. ${ }^{5}$ Anatomy and Developmental Biology Program, Faculty of Medicine, University of Chile, Santiago, Chile.

Received: 14 October 2014 Accepted: 7 January 2015

Published online: 23 January 2015

\section{References}

1. Farías JG, Jiménez D, Osorio J, Zepeda AB, Figueroa CA, Pulgar VM. Acclimatization to chronic intermittent hypoxia in mine workers: a challenge to mountain medicine in Chile. Biol Res. 2013;46:59-67.

2. Bigham AW, Mao X, Mei R, Brutsaert T, Wilson MJ, Julian CG, et al. Identifying positive selection candidate loci for high-altitude adaptation in Andean populations. Hum Genomics. 2009;4:79-90.

3. Caramelo C, Peña JJ, Castilla A, Justo S, De Solis AJ, Neria F, et al. Response to hypoxia. A systemic mechanism based on the control of gene expression. Medicina. 2006;66:155-64.

4. Farías JG, Zepeda AB, Calaf GM. Melatonin protects the heart, lungs and kidneys from oxidative stress under intermittent hypobaric hypoxia in rats. Biol Res. 2012;45:81-5.

5. Schofield CJ, Ratcliff PJ. Oxygen sensing by HIF hydroxylases. Nat Rev Mol Cell Biol. 2004:5:343-54.

6. Sinha S, Dutta A, Singh SN, Ray US. Protein nitration, lipid peroxidation and DNA damage at high altitude in acclimatized lowlanders and native highlanders: relation with oxygen consumption. Respir Physiol Neurobiol. 2010;171:115-21.

7. Magalhaes J, Ascensao A, Soares JMC, Ferreira R, Neuparth MJ, Marques F, et al. Acute and severe hypobaric hypoxia increases oxidative stress and impairs mitochondrial function in mouse skeletal muscle. J Appl Physiol. 2005;99:1247-53.

8. Alvarez P, Tapia L, Mardones LA, Pedemonte JC, Farías JG, Castillo RL. Cellular mechanisms against ischemia reperfusion injury induced by the use of anesthetic pharmacological agents. Chem Biol Interact. 2014;218:89-98.

9. Farias JG, Bustos-Obregón E, Orellana R, Bucarey JL, Quiroz E, Reyes JG. Effects of chronic hypobaric hypoxia on testis histology and round spermatid oxidative metabolism. Andrologia. 2005;37:47-52.

10. Farias JG, Puebla M, Acevedo A, Tapia PJ, Gutierrez E, Zepeda A, et al. Oxidative stress in rat testis and epididymis under intermittent hypobaric hypoxia: protective role of ascorbate supplementation. J Androl. 2010;31:314-21.

11. Hemadi M, Shokri S, Moramezi F, Nikbakht R, Sobhani A. Potential use of melatonin supplementation to protect vitrified testicular grafts from hypoxic-ischaemic damage. Andrologia. 2014;46:513-21.

12. Yang BC, Saldeen TG, Bryant JL, Nichols WW, Mehta JL. Long-polyunsaturated dietary fish oil supplementation protects against ischemia-reperfusioninduced myocardial dysfunction in isolated rat hearts. Am Heart J. 1993;126:1287-92. 
13. Sergiel JP, Martine L, Raederstorff D, Grynberg A, Demaison L. Individual effects of dietary EPA and DHA on the functioning of the isolated working rat heart. Can J Physiol Pharmacol. 1998;76:728-36.

14. Rodrigo R, Korantzopoulos P, Cereceda M, Asenjo R, Zamorano J, Villalabeitia $\mathrm{E}$, et al. A randomized controlled trial to prevent post-operative atrial fibrillation by antioxidant reinforcement. J Am Coll Cardiol. 2013;62:1457-65.

15. Weber $P$, Raederstorff $D$. Triglyceride-lowering effect of omega-3 peroxidation may be key steps in the effect of EPA. LC-polyunsaturated fatty acids - a review. Nutr Metab Cardiovasc Dis. 2000;10:28-37.

16. Weylandt KH, Chiu CY, Gomolka B, Waechter SF, Wiedenmann B. Omega-3 fatty acids and their lipid mediators: towards an understanding of resolvin and protectin formation. Prostaglandins Other Lipid Mediat. 2012:97:73-82.

17. Mori TA, Watts GF, Burke V, Hilme E, Puddey IB, Beilin LJ. Differential effects of eicosapentaenoic acid and docosahexaenoic acid on forearm vascular reactivity of the microcirculation in hyperlipidaemic, overweight men. Circulation. 2000;102:1264-9.

18. Feng GM, Chen JH, Lin Cl, Yang JM. Effect of docosahexaenoic acid on hypoxia/reoxygenation injury in human coronary arterial smooth muscle cells. Eur J Nutr. 2012;51:987-95.

19. Leaf A, Xiao YF, Kang JX, Billman GE. Membrane effects of the $n-3$ fish oil fatty acids, which prevent fatal ventricular arrhythmias. J Membr Biol. 2005;206:129-39.

20. Taltavull N, Muñoz-Cortés M, Lluís L, Jové M, Fortuño A, Molinar-Toribio E, et al. Eicosapentaenoic acid/docosahexaenoic acid 1:1 ratio improves histological alterations in obese rats with metabolic syndrome. Lipids Health Dis. 2014;13:31.

21. Ishikado A, Morino K, Nishio Y, Nakagawa F, Mukose A, Sono Y, et al. 4-Hydroxy hexenal derived from docosahexaenoic acid protects endothelial cells via Nrf2 activation. PLoS One. 2013;8:e69415.

22. Uygur R, Aktas C, Tulubas F, Uygur E, Kanter M, Erboga M, et al. Protective effects of fish omega-3 fatty acids on doxorubicin-induced testicular apoptosis and oxidative damage in rats. Andrologia. 2014;46:917-26.

23. Benzie IF, Strain JJ. The ferric reducing ability of plasma (FRAP) as a measure of "antioxidant power": the FRAP assay. Anal Biochem. 1996;239:70-6.

24. Young IS, Trimble ER. Measurement of malondialdehyde in plasma by high performance liquid chromatography with fluorimetric detection. Ann Clin Biochem. 1991;28:504-8.

25. Nebot C, Moutet M, Huet P, Xu J, Yadan JC, Chaudiere J. Spectrophotometric assay of superoxide dismutase activity based on the activated autoxidation of a tetracyclic catechol. Anal Biochem. 1993;14:442-51.

26. Flohé L, Günzler WA. Assays of glutathione peroxidase. Methods Enzymol. 1984;105:114-21.

27. Hissin PJ, Hilf R. A fluorometric method for determination of oxidized and reduced glutathione in tissues. Anal Biochem. 1976;74:214-26.

28. Ohkawa H, Ohishi N, Yagi K. Assay for lipid peroxides in animal tissues by thiobarbituric acid reaction. Anal Biochem. 1979;95:351-8.

29. Sluiter I, van Heijst A, Haasdijk R, Kempen MB, Boerema-de Munck A, Reiss I, et al. Reversal of pulmonary vascular remodeling in pulmonary hypertensive rats. Exp Mol Pathol. 2012;93:66-73.

30. Jefferson JA, Simoni J, Escudero E, Hurtado ME, Swenson ER, Wesson DE, et al. Increased oxidative stress following acute and chronic high altitude exposure. High Alt Med Biol. 2004;5:61-9.

31. Pichler Hefti J, Sonntag D, Hefti U, Risch L, Schoch OD, Turk AJ, et al. Oxidative stress in hypobaric hypoxia and influence on vessel-tone modifying mediators. High Alt Med Biol. 2013;14:273-9.

32. Ballmann C, McGinnis G, Peters B, Slivka D, Cuddy J, Hailes W, et al. Exercise-induced oxidative stress and hypoxic exercise recovery. Eur J Appl Physiol. 2014;114:725-33.

33. McGinnis G, Kliszczewiscz B, Barberio M, Ballmann C, Peters B, Slivka D, et al. Acute Hypoxia and Exercise-Induced Blood Oxidative Stress. Int J Sport Nutr Exerc Metab. 2014. in press.

34. Maiti P, Singh SB, Sharma AK, Muthuraju S, Banerjee PK, llavazhagan G. Hypobaric hypoxia induces oxidative stress in rat brain. Neurochem Int. 2006:49:709-16.

35. Lopez V, Siques P, Brito J, Vallejos C, Naveas N, Carvallo C, et al. Upregulation of arginase expression and activity in hypertensive rats exposed to chronic intermittent hypobaric hypoxia. High Alt Med Biol. 2009;10:373-81.

36. Zepeda AB, Figueroa CA, Calaf GM, Farias JG. Male reproductive system and antioxidants in oxidative stress induced by hypobaric hypoxia. Andrologia. 2014;46(1):1-8

37. Giaccia AJ, Simon MC, Johnson R. The biology of hypoxia: the role of oxygen sensing in development, normal function, and disease. Genes Dev. 2004;18:2183-94.

38. Irwin DC, McCord JM, Nozik-Grayck E, Beckly G, Foreman B, Sullivan T, et al. A potential role for reactive oxygen species and the HIF-1alpha-VEGF pathway in hypoxiainduced pulmonary vascular leak. Free Radic Biol Med. 2009:47:55-61.

39. Zepeda AB, Pessoa Jr A, Castillo RL, Figueroa CA, Pulgar VM, Farías JG. Cellular and molecular mechanisms in the hypoxic tissue: role of HIF-1 and ROS. Cell Biochem Funct. 2013;31:451-9.

40. Siervo M, Riley HL, Fernandez BO, Leckstrom CA, Martin DS, Mitchell K, et al. Effects of prolonged exposure to hypobaric hypoxia on oxidative stress, inflammation and gluco-insular regulation: the not-so-sweet price for good regulation. PLoS One. 2014;9:e94915.

41. Zhang W, Hu X, Yang W, Gao Y, Chen J. Omega-3 polyunsaturated fatty acid supplementation confers long-term neuroprotection against neonatal hypoxic-ischemic brain injury through anti-inflammatory actions. Stroke. 2010;41:2341-7.

42. Adkins $Y$, Kelley DS. Mechanisms underlying the cardioprotective effects of omega-3 polyunsaturated fatty acids. J Nutr Biochem. 2010;21:781-92.

43. Miyata T, Takizawa S, Van Ypersele de $S$ trihou C. Intracellular sensors for oxygen and oxidative stress: novel therapeutic targets. Am J Physiol Cell Physiol. 2011;300:C226-31.

44. Zhu Y, Yan Y, Gius DR, Vassilopoulos A. Metabolic regulation of Sirtuins upon fasting and the implication for cancer. Curr Opin Oncol. 2013:25:630-6.

45. Pardo PS, Mohamed JS, Lopez MA, Boriek AM. Induction of Sirt1 by mechanical stretch of skeletal muscle through the early response factor EGR1 triggers an antioxidative response. J Biol Chem. 2011;286:2559-66.

46. Cheng $Y$, Takeuchi $H$, Sonobe $Y$, Jin S, Wang $Y$, Horiuchi $H$, et al. Sirtuin 1 attenuates oxidative stress via upregulation of superoxide dismutase 2 and catalase in astrocytes. J Neuroimmunol. 2014;269:38-43.

47. Xue B, Yang Z, Wang X, Shi H. Omega-3 polyunsaturated fatty acids antagonize macrophage inflammation via activation of AMPK/SIRT1 pathway. PLoS One. 2012;7:e45990.

48. Jing $H$, Yao J, Liu X, Fan H, Zhang F, Li Z, et al. Fish-oil emulsion (omega-3 polyunsaturated fatty acids) attenuates acute lung injury induced by intestinal ischemia-reperfusion through Adenosine $5^{\prime}$-monophosphate-activated protein kinase-sirtuin1 pathway. J Surg Res. 2014;187:252-61.

49. Castillo RL, Arias C, Farías JG. Omega 3 chronic supplementation attenuates myocardial ischaemia-reperfusion injury through reinforcement of antioxidant defense system in rats. Cell Biochem Funct. 2014;32:274-81.

50. Kubo K, Saito M, Tadokoro T, Maekawa A. Preferential incorporation of docosahexaenoic acid into nonphosphorus lipids and phosphatidylethanolamine protects rats from dietary DHA-stimulated lipid peroxidation. J Nutr. 2000;130:1749-59.

51. Gurzell EA, Wiesinger JA, Morkam C, Hemmrich S, Harris WS, Fenton JI. Is the omega-3 index a valid marker of intestinal membrane phospholipid $\mathrm{EPA}+\mathrm{DHA}$ content? Prostaglandins Leukot Essent Fatty Acids. 2014:91:87-96.

52. Pantazi E, Zaouali MA, Bejaoui M, Serafin A, Folch-Puy E, Petegnief $V$, et al. Silent information regulator 1 protects the liver against ischemia-reperfusion injury: implications in steatotic liver ischemic preconditioning. Transpl Int. 2014;27:493-503. 